\title{
Uniform Extendibility of the Bergman Kernel for Generalized Minimal Balls
}

\begin{abstract}
Jong-Do Park
Department of Mathematics and Research Institute for Basic Sciences, Kyung Hee University, Seoul 130-701, Republic of Korea
\end{abstract}

Correspondence should be addressed to Jong-Do Park; mathjdpark@khu.ac.kr

Received 6 October 2014; Accepted 23 December 2014

Academic Editor: Kehe Zhu

Copyright (C) 2015 Jong-Do Park. This is an open access article distributed under the Creative Commons Attribution License, which permits unrestricted use, distribution, and reproduction in any medium, provided the original work is properly cited.

We consider a class of convex domains which contains non-Reinhardt domains with nonsmooth boundary. We show that the domains of this class satisfy the condition $\mathbf{Q}$.

\section{Introduction}

Let $\Omega$ be a bounded domain in $\mathbb{C}^{n}$. The Bergman space $A^{2}(\Omega)$ is the space of holomorphic functions on $\Omega$ which are square integrable. The Bergman space has the Bergman kernel $K(z, w)$, which is uniquely determined by the properties that it is an element of $A^{2}(\Omega)$ in $z$, is conjugate symmetric, and reproduces $A^{2}(\Omega)$. The Bergman projection $P$ of $L^{2}(\Omega)$ onto $A^{2}(\Omega)$ is given by

$$
(P f)(w)=\int_{\Omega} f(z) \overline{K(z, w)} d v(z),
$$

where $f \in L^{2}(\Omega)$ and $w \in \Omega$.

A bounded domain $\Omega$ is said to satisfy the condition $\mathbf{Q}$ if the Bergman projection maps $C_{0}^{\infty}(\Omega)$ into the space $\mathcal{O}(\bar{\Omega})$ of all holomorphic functions on $\Omega$ that can be extended holomorphically to a neighborhood of $\bar{\Omega}$.

It was also known that the condition $\mathbf{Q}$ holds on a domain $\Omega$ whenever the $\bar{\partial}$-Neumann problem is globally real analytic hypoelliptic on $\Omega$ [1-3]. When a domain is a smoothly bounded pseudoconvex domain with real analytic boundary, there are many well-known examples on which the globally real analytic hypoellipticity of the $\bar{\partial}$-Neumann problem holds:

(i) strictly pseudoconvex domains [4-6],

(ii) smooth bounded pseudoconvex circular domains with $\sum_{j=1}^{n} z_{j}\left(\partial r / \partial z_{j}\right) \neq 0$ near the boundary, where $r(z)$ is the defining function for domains [7],

(iii) Reinhardt domains [8].
In this paper, we consider a class of convex circular domains which neither are Reinhardt nor have smooth boundaries and prove that the condition $\mathbf{Q}$ holds on each domain in the class defined above. Recently, there are many results of computing the Bergman kernels for various domains explicitly. See [9-14].

Let us restrict our attention to circular domains which are not Reinhardt. For $z=\left(z_{1}, \ldots, z_{n}\right) \in \mathbb{C}^{n}$, consider the norm

$$
N_{*}(z)=\sqrt{\frac{|z|^{2}+|z \cdot z|}{2}},
$$

where $z \cdot w=\sum_{j=1}^{n} z_{j} w_{j}$ and $|z|^{2}=z \cdot \bar{z}$. The norm $N_{*}$ is the smallest norm in $\mathbb{C}^{n}$ that coincides with the Euclidean norm in $\mathbb{R}^{n}$, under certain restriction [15]. The minimal ball $\mathbb{B}_{*}:=\left\{z \in \mathbb{C}^{n}: N_{*}(z)<1\right\}$ is the first known bounded domain in $\mathbb{C}^{n}$ which is neither Reinhardt nor homogeneous and for which the Bergman kernel can be computed explicitly as the closed form [16].

For any positive integers $p$ and $q$, we denote by $M_{p, q}(\mathbb{C})$ the $p q$-dimensional complex vector space of all $(p \times q)$ matrices with complex coefficients. If $Z=\left(z_{j k}\right)_{1 \leq j \leq p ; 1 \leq k \leq q}$ is an element of $M_{p, q}(\mathbb{C})$, we set

$$
\|Z\|_{*}:=\left\{\sum_{j=1}^{p}\left(\sum_{k=1}^{q}\left|z_{j k}\right|^{2}+\left|\sum_{k=1}^{q} z_{j k}^{2}\right|\right)\right\}^{1 / 2} .
$$

Let $d$ be a positive integer and let $m=\left(m_{1}, \ldots, m_{d}\right)$ and $n=$ $\left(n_{1}, \ldots, n_{d}\right)$ be multi-indices with $m_{j} \geq 1$ and $n_{j} \geq 1$ for all 
$j=1, \ldots, d$. We denote by $j_{0}$ the number of those $n_{j}$ 's that are equal to 1 . If $j_{0} \geq 1$, we may assume without loss of generality that $n_{1}=\cdots=n_{j_{0}}=1$ and $n_{j} \geq 2$ for $j=j_{0}+1, \ldots, d$. Let $a=\left(a_{1}, \ldots, a_{d}\right) \in \mathbb{R}^{d}$ with $a_{j} \geq 1$, and consider the function $\rho_{a, m, n}(Z)$ defined for $Z=(Z(1), \ldots, Z(d)) \in M_{m_{1}, n_{1}}(\mathbb{C}) \times \cdots \times$ $M_{m_{d}, n_{d}}(\mathbb{C})$ by

$$
\rho_{a, m, n}(Z):=\sum_{j=1}^{d}\|Z(j)\|_{*}^{2 a_{j}}
$$

and define the generalized minimal balls $\Omega_{a, m, n}:=\{Z$ : $\left.\rho_{a, m, n}(Z)<1\right\}$, where

$$
\begin{gathered}
Z(j):=\left(\begin{array}{c}
Z_{1}(j) \\
\vdots \\
Z_{m_{j}}(j)
\end{array}\right), \\
Z_{l}(j):=\left(Z_{l, 1}(j), \ldots, Z_{l, n_{j}}(j)\right) \in \mathbb{C}^{n_{j}} .
\end{gathered}
$$

Note that

$$
\begin{aligned}
\|Z(j)\|_{*} & =\sqrt{\sum_{l=1}^{m_{j}}\left(\sum_{r=1}^{n_{j}}\left|Z_{l, r}(j)\right|^{2}+\left|\sum_{r=1}^{n_{j}} Z_{l, r}(j)^{2}\right|\right)} \\
& =\sqrt{\sum_{l=1}^{m_{j}} N\left(Z_{l}(j)\right)^{2}},
\end{aligned}
$$

where $N(\cdot):=\sqrt{2} N_{*}(\cdot)$.

Remark 1. (i) Note that, for $n=(1, \ldots, 1)$, the domain $\Omega_{a, m, n}$ is a complex ellipsoid, which is a smoothly bounded Reinhardt domain and clearly satisfies the condition $\mathbf{Q}$ [8].

(ii) Note also that, for $d=1$ and $a=m=(1)$, the domain $\Omega_{a, m, n}$ is the minimal ball with radius $1 / \sqrt{2}$, which is not Reinhardt but satisfies the condition $\mathbf{Q}$ [16]. If at least one of $n_{j}$ 's is greater than 1 , then $\Omega_{a, m, n}$ is neither smooth nor Reinhardt.

In 2002, Youssfi [17] computed the Bergman kernel for the generalized minimal ball $\Omega_{a, m, n}$ using the transformation formula of the Bergman kernel under proper holomorphic mappings [18-20]. See the Bergman kernel for $\Omega_{a, m, n}$ in Theorem 4 . The Bergman kernel for $\Omega_{a, m, n}$ contains an infinite series $\mathscr{H}_{a, m, n}(x)$ defined by

$$
\begin{gathered}
\sum_{k_{1}=0}^{\infty} \cdots \sum_{k_{d}=0}^{\infty}\left(\left(\Gamma \left(1+\sum_{j=1}^{j_{0}} \frac{k_{j}+m_{j}}{a_{j}}\right.\right.\right. \\
\left.\left.+\sum_{j=j_{0}+1}^{d} \frac{k_{j}+m_{j} n_{j}-m_{j}}{a_{j}}\right)\right) \\
\cdot\left(\prod_{j=1}^{j_{0}} \Gamma\left(\frac{k_{j}+m_{j}}{a_{j}}\right)\right.
\end{gathered}
$$

$$
\begin{gathered}
\left.\left.\cdot \prod_{j=j_{0}+1}^{d} \Gamma\left(\frac{k_{j}+m_{j} n_{j}-m_{j}}{a_{j}}\right)\right)^{-1}\right) \\
\cdot x_{1}^{k_{1}} \cdots x_{d}^{k_{d}} .
\end{gathered}
$$

It is the crucial part to investigate the infinite series $\mathscr{H}_{a, m, n}(x)$, when we study the Bergman kernel for $\Omega_{a, m, n}$.

In [16], it was proved that the minimal ball satisfies the condition $\mathbf{Q}$. In this paper, we generalize this result to the generalized minimal balls $\Omega_{a, m, n}$ as follows.

Theorem 2. The condition $\mathbf{Q}$ holds on each domain

$$
\Omega_{a, m, n}=\left\{Z: \rho_{a, m, n}(Z)<1\right\}
$$

where $\rho_{a, m, n}(Z)$ is defined as in (4) and all $a_{j}$ 's are positive integers.

Similarly as in [16], we obtain the properties of the proper holomorphic mappings on $\Omega_{a, m, n}$.

Corollary 3. Let $D \subset \mathbb{C}^{m_{1} n_{1}+\cdots+m_{d} n_{d}}$ be any bounded circular domain which contains the origin.

(i) If $f: \Omega_{a, m, n} \rightarrow D$ is a proper holomorphic mapping, then $f$ extends holomorphically to a neighborhood of $\bar{\Omega}_{a, m, n}$.

(ii) If $D$ is smooth then there is no proper holomorphic mapping from $\Omega_{a, m, n}$ into $D$.

In Section 2, we review the notation and the Bergman kernel for $\Omega_{a, m, n}$ following [17]. In Section 3, we prove the theorem using the characterization theorem (Lemma 6). In the final section, the explicit formulas of $\mathscr{H}_{a, m, n}(x)$ have been obtained in some cases and we show that the minimal ball satisfies the condition $\mathbf{Q}$ using Theorem 2.

\section{Bergman Kernel Function for $\Omega_{a, m, n}$}

In this section we review the Bergman kernel for $\Omega_{a, m, n}$ following the notations which were introduced in [17]. For $u=\left(u_{j, l}\right)_{l=1, \ldots, m_{j} ; j=1, \ldots, d}$ with $u_{j, l} \in \mathbb{C}$, we define

$$
t u:=\left(t_{j, l} u_{j, l}\right)_{l=1, \ldots, m_{j} ; j=1, \ldots, d}, \quad \text { set } t^{2}=t t
$$

Consider the differential operator $\mathscr{D}=\mathscr{D}(a, m, n)$ acting on functions $f(t)$ for $t=\left(t_{j, l}\right)$ by

$$
\begin{aligned}
& (\mathscr{D} f)(t) \\
& \quad:=a_{1} \cdots a_{d}\left(\mathscr{D}_{1,1} \cdots \mathscr{D}_{1, m_{1}}\right) \cdots\left(\mathscr{D}_{d, 1} \cdots \mathscr{D}_{d, m_{d}}\right)(f(t)),
\end{aligned}
$$


where

$$
\begin{aligned}
& \left(\mathscr{D}_{j, l} f\right)(t) \\
& \quad:= \begin{cases}4 f(t) & \text { if } j \leq j_{0}, \\
\frac{2}{\left(n_{j}-1\right) !}\left(2 t_{j, l} \frac{d f}{d t_{j, l}}+\left(n_{j}-1\right) f(t)\right) & \text { if } j>j_{0} .\end{cases}
\end{aligned}
$$

We also consider the differential operator $\widetilde{\mathscr{D}}=\widetilde{\mathscr{D}}(a, m, n)$ acting on functions $g(x)$ for $x=\left(x_{1}, \ldots, x_{d}\right)$ defined by

$$
(\widetilde{\mathscr{D}} g)(x):=\widetilde{\mathscr{D}}_{1} \ldots \widetilde{\mathscr{D}}_{d}\left(\prod_{j=1}^{j_{0}} x_{j}^{m_{j}-1} \prod_{j=j_{0}+1}^{d} x_{j}^{m_{j} n_{j}-m_{j}-1} g(x)\right),
$$

where

$$
\widetilde{D}_{j}:= \begin{cases}\frac{d^{m_{j}-1}}{d x_{j}^{m_{j}-1}} & \text { if } j \leq j_{0}, \\ \frac{d^{m_{j} n_{j}-m_{j}-1}}{d x_{j}^{m_{j} n_{j}-m_{j}-1}} & \text { if } j>j_{0} .\end{cases}
$$

Note that $j_{0}$ depends on $n$. Since $n_{j} \geq 2$ for $j>j_{0}$, then we have $m_{j} n_{j}-m_{j}-1 \geq m_{j}-1 \geq 0$.

We also consider the product group

$$
\Lambda:=\{-1,1\}^{m_{j_{0}+1}} \times \cdots \times\{-1,1\}^{m_{d}}
$$

and extend each element $\varepsilon=\left(\varepsilon_{j, l}\right), l=1, \ldots, m_{j}, j=j_{0}+$ $1, \ldots, d$, of $\Lambda$ to an element $\widetilde{\varepsilon}$ of $\{-1,1\}^{m}$ by

$$
\widetilde{\varepsilon}_{j, l}:= \begin{cases}1 & \text { if } j \leq j_{0}, \\ \varepsilon_{j, l} & \text { if } j>j_{0} .\end{cases}
$$

If $f(t)$ is a smooth function defined on the region of $t=$ $\left(t_{j, l}\right)$ with $\sum_{j=1}^{d} \sum_{l=1}^{m_{j}}\left|t_{j, l}\right|<1$, we consider the function $\tilde{f}$ on the region of $(t, u)$ with $\sum_{j=1}^{d} \sum_{l=1}^{m_{j}}\left(\left|t_{j, l}\right|+\left|u_{j, l}\right|\right)<1$ in $\mathbb{C}^{m} \times \mathbb{C}^{m}$ by

$$
\widetilde{f}(t, u):=\sum_{\varepsilon \in \Lambda} \frac{f(t+\tilde{\varepsilon} u)}{\prod_{j=j_{0}+1}^{d} \prod_{l=1}^{m_{j}} \varepsilon_{j, l} u_{j, l}},
$$

where $\mathbb{C}^{m}=\mathbb{C}^{m_{1}} \times \cdots \times \mathbb{C}^{m_{d}}$. Then define $\mathscr{L} f(t, u):=$ $\tilde{f}(t, \sqrt{u})$. For example, we consider $d=2$ and $j_{0}=1$. Then,

$$
\begin{aligned}
\tilde{f}(t, u)= & \sum_{\varepsilon \in \Lambda} \frac{f\left(t_{1,1}+u_{1,1}, t_{2,1}+\varepsilon_{2,1} u_{2,1}\right)}{\varepsilon_{2,1} u_{2,1}} \\
= & \frac{f\left(t_{1,1}+u_{1,1}, t_{2,1}+u_{2,1}\right)}{u_{2,1}} \\
& -\frac{f\left(t_{1,1}+u_{1,1}, t_{2,1}-u_{2,1}\right)}{u_{2,1}} .
\end{aligned}
$$

Consider the series defined for $x=\left(x_{1}, \ldots, x_{d}\right) \in \mathbb{C}^{d}$ by

$$
G(x):=\widetilde{\mathscr{D}}\left(\mathscr{H}_{a, m, n}\right)(x),
$$

where $\mathscr{H}_{a, m, n}(x)$ is defined in (7). The series $G(x)$ converges for $|x|<1$. Finally, we set

$$
\begin{gathered}
R(t):=G\left(\sum_{l=1}^{m_{1}} t_{1, l}, \ldots, \sum_{l=1}^{m_{d}} t_{d, l}\right), \\
H(t, u):=(\mathscr{L}(\mathscr{D} R))(t, u) .
\end{gathered}
$$

We extend the operation $\bullet$ to a $\mathbb{C}^{m}$-valued bilinear mapping defined for $Z, W \in M_{m_{1}, n_{1}}(\mathbb{C}) \times \cdots \times M_{m_{d}, n_{d}}(\mathbb{C})$ by

$$
Z \bullet W:=\left(Z_{l}(j) \bullet W_{l}(j)\right)_{l=1, \ldots, m_{j} ; j=1, \ldots, d}
$$

if $Z=(Z(1), \ldots, Z(d)), W=(W(1), \ldots, W(d))$, and

$$
Z(j)=\left(\begin{array}{c}
Z_{1}(j) \\
\vdots \\
Z_{m_{j}}(j)
\end{array}\right), \quad W(j)=\left(\begin{array}{c}
W_{1}(j) \\
\vdots \\
W_{m_{j}}(j)
\end{array}\right) .
$$

In 2002, Youssfi [17] computed the Bergman kernel for $\Omega_{a, m, n}$ as follows.

Theorem 4 ([17], Theorem A). The Bergman kernel function for the domain $\Omega=\Omega_{a, m, n}$ is given by the formula

$$
K_{\Omega}(Z, W)=\frac{1}{v(\Omega)} \cdot \frac{H(t, u)}{H(0,0)}, \quad \text { for } Z, W \in \Omega,
$$

where $t=Z \bullet \bar{W}$ and $u=(Z \bullet Z)(\overline{W \bullet W})$. The latter product is understood in the sense of (9). More precisely, one has $t=$ $\left(t_{j, l}\right)_{l=1, \ldots, m_{j} ; j=1, \ldots, d}$ and $u=\left(u_{j, l}\right)_{l=1, \ldots, m_{j} ; j=1, \ldots, d}$, where

$$
\begin{aligned}
t_{j, l} & =Z_{l}(j) \cdot \overline{W_{l}(j)}=\sum_{r=1}^{n_{j}} Z_{l, r}(j) \overline{W_{l, r}(j)}, \\
u_{j, l} & =\left(Z_{l}(j) \cdot Z_{l}(j)\right)\left(\overline{W_{l}(j) \bullet W_{l}(j)}\right) \\
& =\sum_{r=1}^{n_{j}} Z_{l, r}(j)^{2} \sum_{r=1}^{n_{j}} \overline{W_{l, r}(j)^{2}} .
\end{aligned}
$$

Remark 5. In fact, the weighted Bergman kernel and Szegö kernel have been obtained in [17].

\section{Proof of Theorem 2}

For the study of $\mathscr{H}_{a, m, n}(x)$, we need to introduce Appell's hypergeometric functions. Recall that Appell's hypergeometric function [21] is defined by

$$
\begin{aligned}
F_{A}^{(n)}\left(\alpha ; \beta_{1}, \ldots, \beta_{n} ; \gamma_{1}, \ldots, \gamma_{n} ; \zeta_{1}, \ldots, \zeta_{n}\right) \\
\quad=\sum_{k_{1}=0}^{\infty} \cdots \sum_{k_{n}=0}^{\infty} \frac{(\alpha)_{k_{1}+\cdots+k_{n}}\left(\beta_{1}\right)_{k_{1}} \cdots\left(\beta_{n}\right)_{k_{n}}}{k_{1} ! \cdots k_{n} !\left(\gamma_{1}\right)_{k_{1}} \cdots\left(\gamma_{n}\right)_{k_{n}}} \zeta_{1}^{k_{1}} \cdots \zeta_{n}^{k_{n}},
\end{aligned}
$$

where $(a)_{m}=\Gamma(a+m) / \Gamma(a)$. 
Now we prove the main theorem of this paper. At first we need to represent $\mathscr{H}_{a, m, n}(x)$ in terms of Appell hypergeometric functions $F_{A}^{(d)}$ when $a_{j}$ 's are positive integers. If we write

$$
h_{j}= \begin{cases}m_{j}, & j \leq j_{0} ; \\ m_{j}\left(n_{j}-1\right), & j>j_{0},\end{cases}
$$

then we have

$$
\begin{aligned}
& \mathscr{H}_{a, m, n}(x) \\
& =\sum_{k_{1}=0}^{\infty} \cdots \sum_{k_{d}=0}^{\infty} \frac{\Gamma\left(1+\sum_{j=1}^{d}\left(\left(k_{j}+h_{j}\right) / a_{j}\right)\right)}{\prod_{j=1}^{d} \Gamma\left(\left(k_{j}+h_{j}\right) / a_{j}\right)} x^{k} \\
& =\prod_{j=1}^{d} x_{j}^{1-h_{j}} \sum_{k_{1}=h_{1}-1}^{\infty} \cdots \sum_{k_{d}=h_{d}-1}^{\infty} \frac{\Gamma\left(1+\sum_{j=1}^{d}\left(\left(k_{j}+1\right) / a_{j}\right)\right)}{\prod_{j=1}^{d} \Gamma\left(\left(k_{j}+1\right) / a_{j}\right)} x^{k} \\
& =\prod_{j=1}^{d} x_{j}^{1-h_{j}}\left(I(a ; x)+P_{0}(x)\right),
\end{aligned}
$$

where $P_{0}(x)=P_{0}\left(x_{1}, \ldots, x_{d}\right)$ is a polynomial such that there exists a $j$ with $1 \leq j \leq d$ satisfying that the degree in $x_{j}$ is strictly less than $h_{j}-1$ and

$$
I(a ; x):=\sum_{k_{1}=0}^{\infty} \cdots \sum_{k_{d}=0}^{\infty} \frac{\Gamma\left(1+\sum_{j=1}^{d}\left(\left(k_{j}+1\right) / a_{j}\right)\right)}{\prod_{j=1}^{d} \Gamma\left(\left(k_{j}+1\right) / a_{j}\right)} x^{k} .
$$

Thus we have

$$
\begin{aligned}
G(x) & =\widetilde{\mathscr{D}}_{1} \ldots \widetilde{\mathscr{D}}_{d}\left(\prod_{j=1}^{d} x_{j}^{h_{j}-1} \mathscr{H}_{a, m, n}(x)\right) \\
& =\frac{\partial^{h_{1}-1}}{\partial x_{1}^{h_{1}-1}} \cdots \frac{\partial^{h_{d}-1}}{\partial x_{d}^{h_{d}-1}} I(a ; x) .
\end{aligned}
$$

If $a_{j}$ 's are positive integers, then there are positive integers $p_{j}$ and $q_{j}$ such that $k_{j}=a_{j} p_{j}+q_{j}$ and $0 \leq q_{j} \leq a_{j}-1$. It follows that

$I(a ; x)$

$$
\begin{aligned}
=\sum_{q_{1}=0}^{a_{1}-1} \cdots \sum_{q_{d}=0}^{a_{d}-1} \sum_{p_{1}=0}^{\infty} \cdots \sum_{p_{d}=0}^{\infty}\left(\left(\Gamma \left(1+\sum_{j=1}^{d} p_{j}\right.\right.\right. \\
\left.\left.+\sum_{j=1}^{d} \frac{q_{j}+1}{a_{j}}\right)\right) \\
\left.\cdot\left(\prod_{j=1}^{d} \Gamma\left(p_{j}+\frac{q_{j}+1}{a_{j}}\right)\right)^{-1}\right)
\end{aligned}
$$

$$
\begin{aligned}
& \cdot \prod_{j=1}^{d} x_{j}^{a_{j} p_{j}+q_{j}} \\
& =\sum_{q_{1}=0}^{a_{1}-1} \ldots \sum_{q_{d}=0}^{a_{d}-1} \prod_{j=1}^{d} c\left(p_{j}, q_{j}, a_{j}\right) x_{j}^{q_{j}} \\
& \times F_{A}^{(d)}\left(1+\sum_{j=1}^{d} p_{j}+\sum_{j=1}^{d} \frac{q_{j}+1}{a_{j}} ;\right. \\
& \left.\mathbf{1} ; \frac{\mathbf{q}+\mathbf{1}}{\mathbf{a}} ; \mathbf{x}^{\mathbf{a}}\right),
\end{aligned}
$$

where

$$
c\left(p_{j}, q_{j}, a_{j}\right):=\frac{\Gamma\left(1+\sum_{j=1}^{d} p_{j}+\sum_{j=1}^{d}\left(\left(q_{j}+1\right) / a_{j}\right)\right)}{\prod_{j=1}^{d} \Gamma\left(\left(q_{j}+1\right) / a_{j}\right)} .
$$

Here we used the notation $\mathbf{1}=(\underbrace{1, \ldots, 1}_{d})$ and $(\mathbf{q}+\mathbf{1}) / \mathbf{a}=\left(\left(q_{1}+\right.\right.$ 1) $\left./ a_{1}, \ldots,\left(q_{d}+1\right) / a_{d}\right)$.

Note that Appell's hypergeometric functions $F_{A}^{(n)}(\alpha ; \beta ; \gamma ; x)$ converge for $|x|<1$ and diverge for $|x|>1$. Thus there exist a nonnegative constant $C_{J}$ and an analytic function $P_{1}(x)$ such that

$$
I(a ; x)=\frac{P_{1}(x)}{\prod_{J \subset\{1, \ldots, d\}}\left(1-\sum_{j \in J} x_{j}^{a_{j}}\right)^{C_{J}}} .
$$

From (28), there exist a nonnegative constant $C_{J}^{*}$ and an analytic function $P_{2}(x)$ such that

$$
G(x)=\frac{P_{2}(x)}{\prod_{J \subset\{1, \ldots, d\}}\left(1-\sum_{j \in J} x_{j}^{\left.a_{j}\right)^{C_{J}^{*}}} .\right.}
$$

From (19), there exists an analytic function $P_{3}(x)$ such that

$$
\mathscr{D} R(t)=\prod_{j=1}^{d}\left(a_{j} \prod_{l=1}^{m_{j}} \mathscr{D}_{j, l}\right) R(t)=\frac{P_{3}(t)}{P_{4}(t)},
$$

where

$$
P_{4}(t):=\prod_{J \subset\{1, . ., d\}}\left(1-\sum_{j \in J}\left(\sum_{l=1}^{m_{j}} t_{j, l}\right)^{a_{j}}\right)^{C_{J}^{*}} .
$$

The following lemma is useful when we show that a bounded domain satisfies the condition $\mathbf{Q}$.

Lemma $6([22,23])$. For a bounded domain $\Omega$, the condition $\mathbf{Q}$ holds on $\Omega$ if and only if, for each compact subset $L$ of $\Omega$, there exists an open set $U_{L}$ containing $\bar{\Omega}$ such that

(i) for each fixed $w_{0} \in \operatorname{LK}\left(z, w_{0}\right)$ is holomorphic in $z$ on $U_{L}$,

(ii) $K(z, w)$ is continuous on $U_{L} \times L$. 
The above property for smoothly bounded domains was proved by Chen [22] and duplicated for general bounded domains by Thomas [23].

At first we need to study the singular points of $\widetilde{f}(t, u)$ defined as in (16).

Lemma 7. Let $f(t)$ be a smooth function on a region of $t=$ $\left(t_{j, l}\right)$ with $\sum_{j=1}^{d} \sum_{l=1}^{m_{j}}\left|t_{j, l}\right|<1$. Denote

$$
P_{f}:=\left\{t=\left(t_{j, l}\right) \in \mathbb{C}^{m}: f(t) \text { has a pole at } t\right\}
$$

If $\widetilde{f}(t, u)$ is defined as in (16), then there exists a function $F(t, u)$ such that

(i) $P_{F}:=\left\{(t, u) \in \mathbb{C}^{m} \times \mathbb{C}^{m}: F(t, u)\right.$ has a pole at $\left.(t, u)\right\}$ equal to $\left\{(t+\varepsilon u) \in P_{f}: \varepsilon \in \Lambda\right\}$,

(ii) $\tilde{f}(t, u)=F(t, u) \times 2^{\sum_{j=j_{0}+1}^{d} m_{j}}$ for $(t, u) \notin P_{F}$.

Proof. If we define $a_{j, l}^{+}=t_{j, l}+u_{j, l}$ and $a_{j, l}^{-}=t_{j, l}-u_{j, l}$, then $t+\widetilde{\varepsilon} u$ is equal to one of

$$
\left(\left(\begin{array}{c}
a_{1,1}^{+} \\
\vdots \\
a_{1, m_{1}}^{+}
\end{array}\right), \ldots,\left(\begin{array}{c}
a_{j_{0}, 1}^{+} \\
\vdots \\
a_{j_{0}, m_{j_{0}}}^{+}
\end{array}\right),\left(\begin{array}{c}
a_{j_{0}+1,1}^{ \pm} \\
\vdots \\
a_{j_{0}+1, m_{j_{0}+1}}^{+}
\end{array}\right), \ldots,\left(\begin{array}{c}
a_{d, 1}^{ \pm} \\
\vdots \\
a_{d, m_{d}}^{ \pm}
\end{array}\right)\right)
$$

where $a_{j, l}^{ \pm}$is one of either $a_{j, l}^{+}$or $a_{j, l}^{-}$.

Let $N_{0}$ be the number of $a_{j, l}^{-}$'s. For fixed $j$ and $l$ with $j_{0}+1 \leq$ $j \leq d, 1 \leq l \leq m_{j}$, we have

$$
\begin{aligned}
\tilde{f}(t, u)= & \frac{1}{\prod_{j=j_{0}+1}^{d} \prod_{l=1}^{m_{j}} u_{j, l}} \sum_{\varepsilon \in \Lambda}(-1)^{N_{0}} f(t+\widetilde{\varepsilon} u) \\
= & \frac{1}{\prod_{j=j_{0}+1}^{d} \prod_{l=1}^{m_{j}} u_{j, l}} \\
& \cdot\left(\sum_{a_{j, l}^{+}}(-1)^{N_{0}} f(t+\widetilde{\varepsilon} u)+\sum_{a_{j, l}^{-}}(-1)^{N_{0}+1} f(t+\widetilde{\varepsilon} u)\right) \\
= & \frac{1}{\prod_{j=j_{0}+1}^{d} \prod_{l=1}^{m_{j}} u_{j, l}} \\
& \cdot\left(\sum_{a_{j, l}^{+}}(-1)^{N_{0}} f(t+\widetilde{\varepsilon} u)-\sum_{a_{j, l}^{-}}(-1)^{N_{0}} f(t+\widetilde{\varepsilon} u)\right),
\end{aligned}
$$

where $\sum_{a_{j, l}^{+}}\left(\sum_{a_{j, l}^{-}}\right.$, resp. $)$means summation running over $\varepsilon \in \Lambda$ which contains $a_{j, l}^{+}\left(\sum_{a_{j, l}^{-}}\right.$, resp.).

From the above formula, we see that a numerator has a factor $a_{j, l}^{+}-a_{j, l}^{-}$. Repeating this process for all $j_{0}+1 \leq j \leq d$ and $1 \leq l \leq m_{j}$, we obtain that $\tilde{f}(t, u)$ is equal to certain function $F(t, u)$ times

$$
\begin{aligned}
\prod_{j=j_{0}+1}^{d} \prod_{l=1}^{m_{j}}\left(a_{j, l}^{+}-a_{j, l}^{-}\right) & =\prod_{j=j_{0}+1}^{d} \prod_{l=1}^{m_{j}} 2 u_{j, l} \\
& =2^{\sum_{j=j_{0}+1}^{d} m_{j}} \prod_{j=j_{0}+1}^{d} \prod_{l=1}^{m_{j}} u_{j, l},
\end{aligned}
$$

which means that $F$ satisfies condition (ii).

Let $t_{j, l}$ and $u_{j, l}$ be defined as in (23). By Lemma 7 the Bergman kernel of $\Omega_{a, m, n}$ has poles at $(t, u) \in \mathbb{C}^{m} \times \mathbb{C}^{m}$ with

$$
\sum_{j \in J} \sum_{l=1}^{m_{j}}\left(t_{j, l} \pm u_{j, l}^{1 / 2}\right)^{a_{j}}=1
$$

for some $J \subset\{1, \ldots, d\}$. Write $t_{j, l}$ and $u_{j, l}$ as

$$
t_{j, l}=\sum_{r=1}^{n_{j}} a_{r} \overline{b_{r}}, \quad u_{j, l}=\sum_{r=1}^{n_{j}} a_{r}^{2} \sum_{r=1}^{n_{j}} \overline{b_{r}^{2}}
$$

where $a_{r}=Z_{l, r}(j)$ and $b_{r}=W_{l, r}(j)$. Then it follows that

$$
\begin{aligned}
& N\left(Z_{l}(j)\right)=\sqrt{\sum_{r=1}^{n_{j}}\left|a_{r}\right|^{2}+\left|\sum_{r=1}^{n_{j}} a_{r}^{2}\right|}, \\
& N\left(W_{l}(j)\right)=\sqrt{\sum_{r=1}^{n_{j}}\left|b_{r}\right|^{2}+\left|\sum_{r=1}^{n_{j}} b_{r}^{2}\right|} .
\end{aligned}
$$

The following lemma is needed when we estimate the function $P_{4}(x)$.

Lemma 8. For $a=\left(a_{1}, \ldots, a_{n}\right)$ and $b=\left(b_{1}, \ldots, b_{n}\right)$ with $a_{r}, b_{r} \in \mathbb{C}$, let

$$
t=\sum_{r=1}^{n} a_{r} \bar{b}_{r}, \quad u=\sum_{r=1}^{n} a_{r}^{2} \cdot \sum_{r=1}^{n} \overline{b_{r}^{2}} .
$$

Then one has

$$
|t \pm \sqrt{u}| \leq \sqrt{\left(\sum\left|a_{r}\right|^{2}+\left|\sum a_{r}^{2}\right|\right)\left(\sum\left|b_{r}\right|^{2}+\left|\sum b_{r}^{2}\right|\right)} .
$$

Proof. By the Schwartz inequality, we have

$$
\begin{aligned}
|t \pm \sqrt{u}| & \leq \sum\left|a_{r} b_{r}\right|+\sqrt{\left|\sum a_{r}^{2} \cdot \sum \overline{b_{r}^{2}}\right|} \\
& \leq \sqrt{\left(\sum\left|a_{r}\right|^{2}\right)\left(\sum\left|b_{r}\right|^{2}\right)}+\sqrt{\left|\sum a_{r}^{2} \cdot \sum \overline{b_{r}^{2}}\right|} \\
& \leq \sqrt{\left(\sum\left|a_{r}\right|^{2}+\left|\sum a_{r}^{2}\right|\right)\left(\sum\left|b_{r}\right|^{2}+\left|\sum b_{r}^{2}\right|\right)} .
\end{aligned}
$$


Then, by using Lemma 8 , the identity (41), and the inequality $(x y)^{(1 / 2) a} \leq(1 / 2)\left(x^{a}+y^{a}\right)$ for $x, y>0$, we have

$$
\begin{aligned}
\mid 1 & -\sum_{j \in J} \sum_{l=1}^{m_{j}}\left(t_{j, l} \pm u_{j, l}^{1 / 2}\right)^{a_{j}} \mid \\
& \geq 1-\sum_{j \in J} \sum_{l=1}^{m_{j}}\left|t_{j, l} \pm u_{j, l}^{1 / 2}\right|^{a_{j}} \\
& \geq 1-\sum_{j \in J} \sum_{l=1}^{m_{j}}\left\{N\left(Z_{l}(j)\right)^{2} \cdot N\left(W_{l}(j)\right)^{2}\right\}^{(1 / 2) a_{j}} \\
& \geq 1-\frac{1}{2} \sum_{j \in J} \sum_{l=1}^{m_{j}} N\left(Z_{l}(j)\right)^{2 a_{j}}+N\left(W_{l}(j)\right)^{2 a_{j}} \\
& =1-\frac{1}{2} \sum_{j \in J}\left(\sum_{l=1}^{m_{j}} N\left(Z_{l}(j)\right)^{2 a_{j}}+\sum_{l=1}^{m_{j}} N\left(W_{l}(j)\right)^{2 a_{j}}\right) \\
& \geq 1-\frac{1}{2} \sum_{j \in J}\left\{\left(\sum_{l=1}^{m_{j}} N\left(Z_{l}(j)\right)^{2}\right)^{a_{j}}+\left(\sum_{l=1}^{m_{j}} N\left(W_{l}(j)\right)^{2}\right)^{a_{j}}\right\} \\
& \geq 1-\frac{1}{2}\{\rho(Z)+\rho(W)\} .
\end{aligned}
$$

Thus by Lemma 6, each domain $\Omega_{a, m, n}$ satisfies the condition Q.

\section{Examples}

In fact, it is difficult to write $\mathscr{H}_{a, m, n}(x)$ explicitly. In the final section, we give some explicit formulas for $\mathscr{H}_{a, m, n}(x)$.

Lemma 9. Let $\alpha=\left(\alpha_{1}, \ldots, \alpha_{n}\right)$ with $\alpha_{j} \in \mathbb{N} \cup\{0\}$ and $t=$ $\left(t_{1}, \ldots, t_{n}\right) \in \mathbb{C}^{n}$. For any $s>0$ and $t \in \mathbb{C}^{n}$ with $\sum_{j=1}^{n}\left|t_{j}\right|<1$, one has

$$
\sum_{\alpha} \frac{\Gamma\left(\sum_{j=1}^{n} \alpha_{j}+s\right)}{\Gamma(s) \Gamma\left(\alpha_{j}+1\right)} \prod_{j=1}^{n} t_{j}^{\alpha_{j}}=\frac{1}{\left(1-\sum_{j=1}^{n} t_{j}\right)^{s}}
$$

Proof. Consider the Taylor expansion of the function in the right hand side of (46). See the details in [11] or [13].

Lemma 10. Let $a(k)$ be a polynomial of degree $r$ with variable $k$. Then there exist unique constants $c_{l}$ for $0 \leq l \leq r$ such that

$$
\sum_{k=0}^{\infty} a(k) x^{k}=\sum_{l=0}^{r} \frac{c_{l}}{(1-x)^{l+1}}
$$

Proof. For a given polynomial $a(k)$, there exist unique constants $b_{l}$ for $1 \leq l \leq r+1$ such that

$$
\begin{gathered}
a(k)=b_{0}+b_{1}(k+1)+b_{2}(k+1)(k+2) \\
+\cdots+b_{r}(k+1) \cdots(k+r) .
\end{gathered}
$$

Note that, for $l \geq 1$,

$$
\begin{aligned}
\frac{1}{(1-x)^{l+1}} & =\frac{1}{l !} \frac{d^{l}}{d x^{l}}\left(\frac{1}{1-x}\right) \\
& =\frac{1}{l !} \frac{d^{l}}{d x^{l}}\left(\sum_{k=0}^{\infty} x^{k}\right) \\
& =\frac{1}{l !} \sum_{k=0}^{\infty}(k+1) \cdots(k+l) x^{k}
\end{aligned}
$$

If we write $c_{l}=b_{l} / l !$, then we obtain (47).

Example 11. The complex $d$-dimensional ball with radius $1 / \sqrt{2}$ : if $d \geq 1$ and $a=m=n=(1, \ldots, 1)$, then using Lemma 9, we have

$$
\mathscr{H}_{a, m, n}(x)=\sum_{h \in \mathbb{N}_{0}^{d}} \frac{1+\sum_{j=1}^{d}\left(h_{j}+1\right)}{\prod_{j=1}^{d} \Gamma\left(h_{j}+1\right)} x^{h}=\frac{\Gamma(d+1)}{\left(1-\sum_{j=1}^{d} x_{j}\right)^{d+1}} .
$$

Example 12. The Thullen domain $\left\{\left(z_{1}, z_{2}\right) \in \mathbb{C}^{2}:\left|z_{1}\right|^{2}+\right.$ $\left.\left|z_{2}\right|^{2 p}<1\right\}$ with a positive integer $p$ : if $d=2, a=(1, p)$, and $n=m=(1,1)$, then

$$
\Omega_{a, m, n}=\left\{\left(z_{1}, z_{2}\right) \in \mathbb{C}^{2}: 2\left|z_{1}\right|^{2}+2^{p}\left|z_{2}\right|^{2 p}<1\right\}
$$

is linearly biholomorphic to the Thullen domain $\left\{\left(z_{1}, z_{2}\right) \in\right.$ $\left.\mathbb{C}^{2}:\left|z_{1}\right|^{2}+\left|z_{2}\right|^{2 p}<1\right\}$. In this case, we have

$$
\mathscr{H}_{a, m, n}(x)=\sum_{h_{1}=0}^{\infty} \sum_{h_{2}=0}^{\infty} \frac{\Gamma\left(2+h_{1}+\left(h_{2}+1\right) / p\right)}{\Gamma\left(h_{1}+1\right) \Gamma\left(\left(h_{2}+1\right) / p\right)} x_{1}^{h_{1}} x_{2}^{h_{2}} .
$$

If we apply Lemma 9 to a variable $x_{1}$, then we obtain

$$
\begin{aligned}
& \mathscr{H}_{a, m, n}(x) \\
& =\sum_{h_{2}=0}^{\infty} \frac{\Gamma\left(2+\left(h_{2}+1\right) / p\right)}{\Gamma\left(\left(h_{2}+1\right) / p\right)} \frac{x_{2}^{h_{2}}}{\left(1-x_{1}\right)^{2+\left(h_{2}+1\right) / p}} \\
& =\sum_{h_{2}=0}^{\infty}\left(\frac{h_{2}+1}{p}\right)\left(1+\frac{h_{2}+1}{p}\right)\left(\frac{x_{2}}{\left(1-x_{1}\right)^{1 / p}}\right)^{h_{2}} \\
& \quad \cdot \frac{1}{\left(1-x_{1}\right)^{2+1 / p}} \\
& =\frac{1}{p^{2}} \sum_{h_{2}=0}^{\infty}\left(h_{2}+1\right)\left(h_{2}+1+p\right)\left(\frac{x_{2}}{\left(1-x_{1}\right)^{1 / p}}\right)^{h_{2}} \\
& \cdot \frac{1}{\left(1-x_{1}\right)^{2+1 / p}} .
\end{aligned}
$$


By Lemma 10, there exist constants $b_{l}$ such that

$$
\begin{aligned}
& \mathscr{H}_{a, m, n}(x) \\
& =\frac{1}{p^{2}} \sum_{h_{2}=0}^{\infty} \sum_{l=0}^{2} \frac{c_{l}}{\left(\left(1-x_{1}\right)^{1 / p}-x_{2}\right)^{l+1}\left(1-x_{1}\right)^{2-l / p}} \\
& =\frac{1}{p^{2}} \sum_{h_{2}=0}^{\infty} \sum_{l=0}^{2} \frac{c_{l} \cdot g_{p}\left(\left(1-x_{1}\right)^{1 / p}, x_{2}\right)}{\left(\left(1-x_{1}\right)-x_{2}^{p}\right)^{l+1}\left(1-x_{1}\right)^{2-l / p}}
\end{aligned}
$$

where $g_{p}\left(x_{1}, x_{2}\right)=\left(x_{1}^{p}-x_{2}^{p}\right) /\left(x_{1}-x_{2}\right)$ is a polynomial for a positive integer $p$. Note that $2-l / p \geq 2-2 / p>0$.

Example 13. A complex ellipsoid $\left\{\left(z_{1}, z_{2}\right) \in \mathbb{C}^{2}:\left|z_{1}\right|^{4}+\right.$ $\left.\left|z_{2}\right|^{4}<1\right\}$ : if $d=2, a=(2,2)$, and $m=n=(1,1)$, then the following formula was proved in [13]:

$$
\begin{aligned}
\mathscr{H}_{a, m, n}(x)= & \frac{x(\pi+2 \arcsin x) f\left(x^{2}, y^{2}\right)}{4 \pi\left(1-x^{2}\right)^{3 / 2}\left(1-x^{2}-y^{2}\right)^{3}} \\
& +\frac{y(\pi+2 \arcsin y) f\left(y^{2}, x^{2}\right)}{4 \pi\left(1-y^{2}\right)^{3 / 2}\left(1-x^{2}-y^{2}\right)^{3}} \\
& +\frac{2 x y}{\left(1-x^{2}-y^{2}\right)^{3}} \\
& +\frac{g\left(x^{2}, y^{2}\right)}{2 \pi\left(1-x^{2}\right)\left(1-y^{2}\right)\left(1-x^{2}-y^{2}\right)^{2}}
\end{aligned}
$$

where $f(a, b)=3-6 a+6 b+3 a^{2}-6 a b-b^{2}$ and $g(a, b)=$ $2-a-b-a^{2}+2 a b-b^{2}$.

Example 14. The minimal ball with radius $1 / \sqrt{2}$ : if $d=1$, $a=m=(1)$, and $n \geq 2$, the infinite series $\mathscr{H}_{a, m, n}(x)$ is equal to

$$
\begin{aligned}
\sum_{h=0}^{\infty} \frac{\Gamma(h+n)}{\Gamma(h+n-1)} x^{h} & =\sum_{h=0}^{\infty}(h+n-1) x^{h} \\
& =\sum_{h=0}^{\infty}(h+1) x^{h}+(n-2) \sum_{h=0}^{\infty} x^{h} \\
& =\frac{(n-1)-(n-2) x}{(1-x)^{2}} \\
& =x^{2-n}\left\{\frac{(n-1) x^{n-2}-(n-2) x^{n-1}}{(1-x)^{2}}\right\} .
\end{aligned}
$$

Since $n \geq 2$, we have $(n-1) x^{n-2}-(n-2) x^{n-1}$ which is analytic in $x$ and this shows that the minimal ball satisfies the condition $\mathbf{Q}$.
Example 15. Let $d=2, m=\left(m_{1}, m_{2}\right)$, and $n=\left(1, n_{2}\right)$ with $n_{2} \geq 2$ and $a=(1,1)$. Then the infinite series $\mathscr{H}_{a, m, n}(x)$ is equal to

$$
\begin{aligned}
& \sum_{h} \frac{\Gamma\left(1+h_{1}+m_{1}+h_{2}+m_{2} n_{2}-m_{2}\right)}{\Gamma\left(h_{1}+m_{1}\right) \Gamma\left(h_{2}+m_{2} n_{2}-m_{2}\right)} x^{h} \\
& =\sum_{h_{1}=0}^{\infty} \frac{x_{1}^{h_{1}}}{\Gamma\left(h_{1}+m_{1}\right)} \\
& \cdot \sum_{h_{2}=0}^{\infty} \frac{\Gamma\left(h_{2}+1+h_{1}+m_{1}+m_{2} n_{2}-m_{2}\right)}{\Gamma\left(h_{2}+m_{2} n_{2}-m_{2}\right)} x_{2}^{h_{2}} \\
& =\sum_{h_{1}=0}^{\infty} \frac{x_{1}^{h_{1}}}{\Gamma\left(h_{1}+m_{1}\right)} x_{2}^{1-m_{2} n_{2}+m_{2}} \\
& \sum_{k_{2}=m_{2} n_{2}-m_{2}-1}^{\infty} \frac{\Gamma\left(k_{2}+2+h_{1}+m_{1}\right)}{\Gamma\left(k_{2}+1\right)} x_{2}^{k_{2}} \\
& =x_{2}^{1-m_{2} n_{2}+m_{2}} \sum_{k_{2}=m_{2} n_{2}-m_{2}-1}^{\infty} \frac{x_{2}^{k_{2}}}{\Gamma\left(k_{2}+1\right)} \\
& \cdot \sum_{h_{1}=0}^{\infty} \frac{\Gamma\left(h_{1}+k_{2}+2+m_{1}\right)}{\Gamma\left(h_{1}+m_{1}\right)} x_{1}^{h_{1}} \\
& =x_{1}^{1-m_{1}} x_{2}^{1-m_{2} n_{2}+m_{2}} \\
& \text {. } \sum_{k_{1}=m_{1}-1}^{\infty} \sum_{k_{2}=m_{2} n_{2}-m_{2}-1}^{\infty} \frac{\Gamma\left(k_{1}+k_{2}+3\right)}{\Gamma\left(k_{1}+1\right) \Gamma\left(k_{2}+1\right)} x_{1}^{k_{1}} x_{2}^{k_{2}} \text {. }
\end{aligned}
$$

Next, for nonnegative integers $l_{1}$ and $l_{2}$, we need to compute the infinite series

$$
\sum_{k_{1}=l_{1}}^{\infty} \sum_{k_{2}=l_{2}}^{\infty} \frac{\Gamma\left(k_{1}+k_{2}+3\right)}{\Gamma\left(k_{1}+1\right) \Gamma\left(k_{2}+1\right)} x_{1}^{k_{1}} x_{2}^{k_{2}} .
$$

It is interesting to compare this series (58) with the left hand side in (46). We can express the infinite series (58) in terms of finite series using Lemma 9. Precisely, the series (58) is equal to

$$
\begin{gathered}
\frac{2}{\left(1-x_{1}-x_{2}\right)^{3}}-\sum_{k_{1}=0}^{l_{1}-1} \frac{x_{1}^{k_{1}}}{\Gamma\left(k_{1}+1\right)} \frac{\Gamma\left(k_{1}+3\right)}{\left(1-x_{2}\right)^{k_{1}+3}} \\
-\sum_{k_{2}=0}^{l_{2}-1} \frac{x_{2}^{k_{2}}}{\Gamma\left(k_{2}+1\right)} \frac{\Gamma\left(k_{2}+3\right)}{\left(1-x_{1}\right)^{k_{2}+3}} \\
+\sum_{k_{1}=0}^{l_{1}-1} \sum_{k_{2}=0}^{l_{2}-1} \frac{\Gamma\left(k_{1}+k_{2}+3\right)}{\Gamma\left(k_{1}+1\right) \Gamma\left(k_{2}+1\right)} x_{1}^{k_{1}} x_{2}^{k_{2}} .
\end{gathered}
$$

\section{Conflict of Interests}

The author declares that there is no conflict of interests regarding the publication of this paper. 


\section{Acknowledgment}

This work was supported by NRF-2010-0011841 from the National Research Foundation of Korea.

\section{References}

[1] S. R. Bell, "Boundary behavior of holomorphic mappings," in Several Complex Variables. Proceedings of the 1981 Hangzhou Conference, pp. 3-6, Birkhäuser, Boston, Mass, USA, 1984.

[2] D. S. Tartakoff, "Local analytic hypoellipticity for square(b) on nondegenerate Cauchy-Riemann manifolds," Proceedings of the National Academy of Sciences of the United States of America, vol. 75, no. 7, pp. 3027-3028, 1978.

[3] D. S. Tartakoff, "The local real analyticity of solutions to b and the $\partial$-Neumann problem," Acta Mathematica, vol. 145, no. 1, pp. 177-204, 1980.

[4] M. Derridj and D. S. Tartakoff, "On the global real analyticity of solutions to the $\partial$-Neumann problem," Communications in Partial Differential Equations, vol. 1, pp. 401-430, 1976.

[5] G. Komatsu, "Global analytic-hypoellipticity of the $\partial$-Neumann problem," Tohoku Mathematical Journal, II Series, vol. 28, no. 1, pp. 145-156, 1976.

[6] F. Treves, "Analytic hypo-ellipticity of a class of pseudodifferential operators with double characteristics and applications to the $\bar{\partial}$-Neumann problem," Communications in Partial Differential Equations, vol. 3, no. 6-7, pp. 475-642, 1978.

[7] S. Chen, "Global analytic hypoellipticity of the $\partial$-Neumann problem on circular domains," Inventiones Mathematicae, vol. 92, pp. 173-185, 1988.

[8] S.-C. Chen, "Global real analyticity of solutions to the $\partial$ Neumann problem on Reinhardt domains," Indiana University Mathematics Journal, vol. 37, no. 2, pp. 421-430, 1988.

[9] H. Ahn and J.-D. Park, "The explicit forms and zeros of the Bergman kernel function for Hartogs type domains," Journal of Functional Analysis, vol. 262, no. 8, pp. 3518-3547, 2012.

[10] J. P. D’Angelo, “A note on the Bergman kernel," Duke Mathematical Journal, vol. 45, no. 2, pp. 259-265, 1978.

[11] J. P. D'Angelo, "An explicit computation of the Bergman kernel function," The Journal of Geometric Analysis, vol. 4, no. 1, pp. 23-34, 1994.

[12] G. Francsics and N. Hanges, "The Bergman kernel of complex ovals and multivariable hypergeometric functions," Journal of Functional Analysis, vol. 142, no. 2, pp. 494-510, 1996.

[13] J.-D. Park, "New formulas of the Bergman kernels for complex ellipsoids in $\mathbb{C}^{2}$," Proceedings of the American Mathematical Society, vol. 136, no. 12, pp. 4211-4221, 2008.

[14] J.-D. Park, "Explicit formulas of the Bergman kernel for 3dimensional complex ellipsoids," Journal of Mathematical Analysis and Applications, vol. 400, no. 2, pp. 664-674, 2013.

[15] K. T. Hahn and P. Pflug, "On a minimal complex norm that extends the real Euclidean norm," Monatshefte für Mathematik, vol. 105, no. 2, pp. 107-112, 1988.

[16] K. Oeljeklaus, P. Pflug, and E. H. Youssfi, “The Bergman kernel of the minimal ball and applications," Annales de l'Institut Fourier, vol. 47, no. 3, pp. 915-928, 1997.

[17] E. H. Youssfi, "Proper holomorphic liftings and new formulas for the Bergman and Szegö kernels," Studia Mathematica, vol. 152, no. 2, pp. 161-186, 2002.
[18] S. R. Bell, "Proper holomorphic mappings and the Bergman projection," Duke Mathematical Journal, vol. 48, no. 1, pp. 167$175,1981$.

[19] S. R. Bell, "The Bergman kernel function and proper holomorphic mappings," Transactions of the American Mathematical Society, vol. 270, no. 2, pp. 685-691, 1982.

[20] S. R. Bell, "Proper holomorphic mappings between circular domains," Commentarii Mathematici Helvetici, vol. 57, no. 4, pp. 532-538, 1982.

[21] P. Appell and J. K. de Fériet, Fonctions hypergéométriques et hypersphériques, Gauthier-Villars, Paris, France, 1926.

[22] S.-C. Chen, "A remark on the uniform extendability of the Bergman kernel function," Mathematische Annalen, vol. 291, no. 1, pp. 481-486, 1991.

[23] A. D. Thomas, "Uniform extendibility of the Bergman kernel," Illinois Journal of Mathematics, vol. 39, no. 4, pp. 598-605, 1995. 


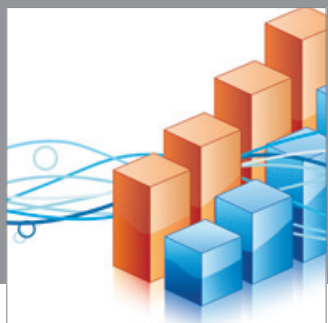

Advances in

Operations Research

mansans

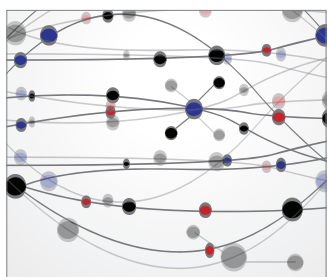

The Scientific World Journal
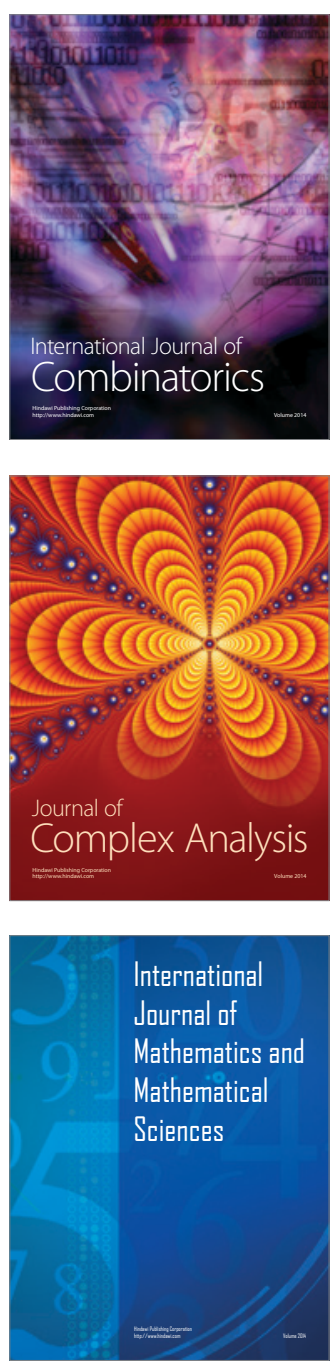
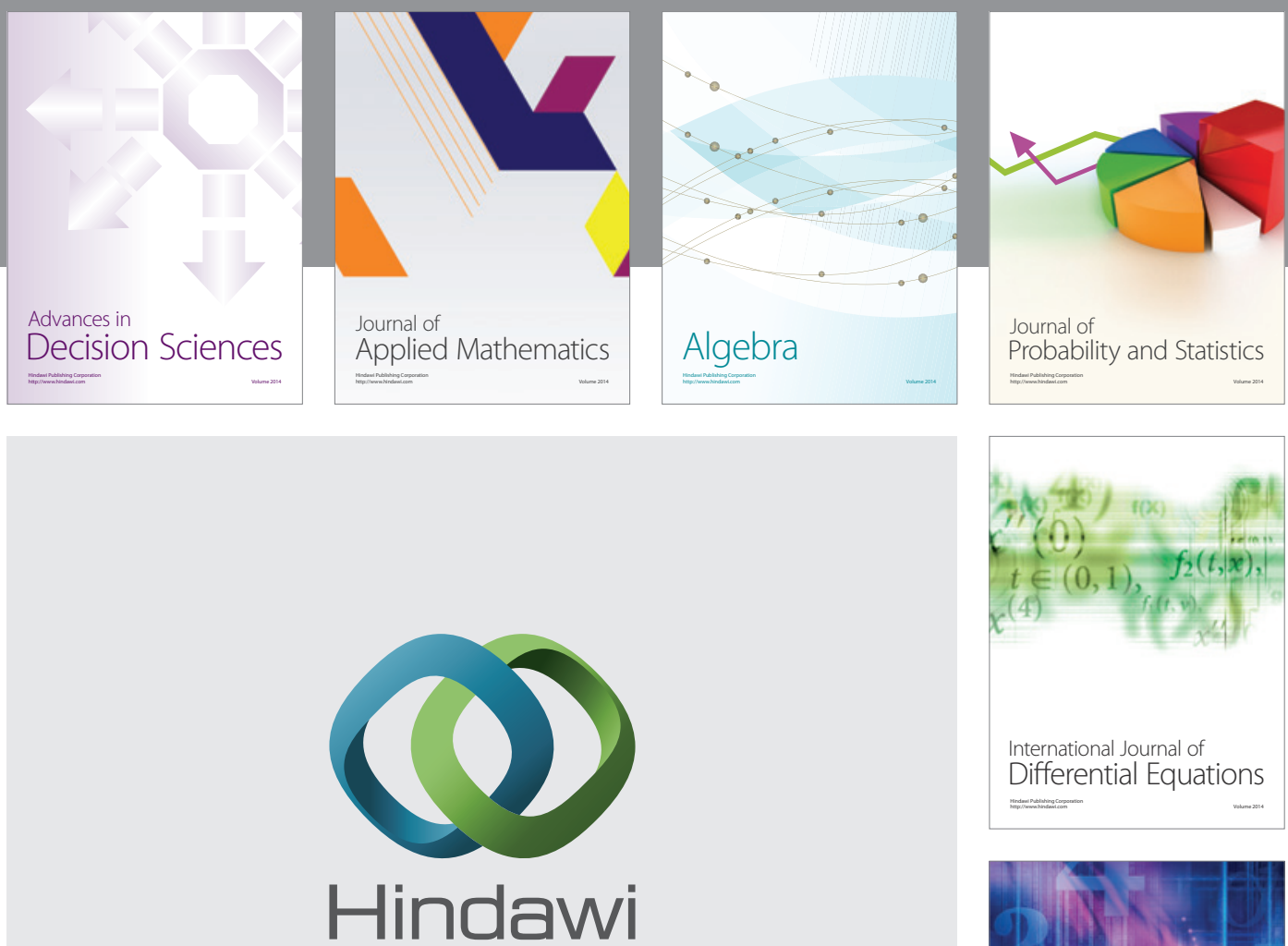

Submit your manuscripts at http://www.hindawi.com
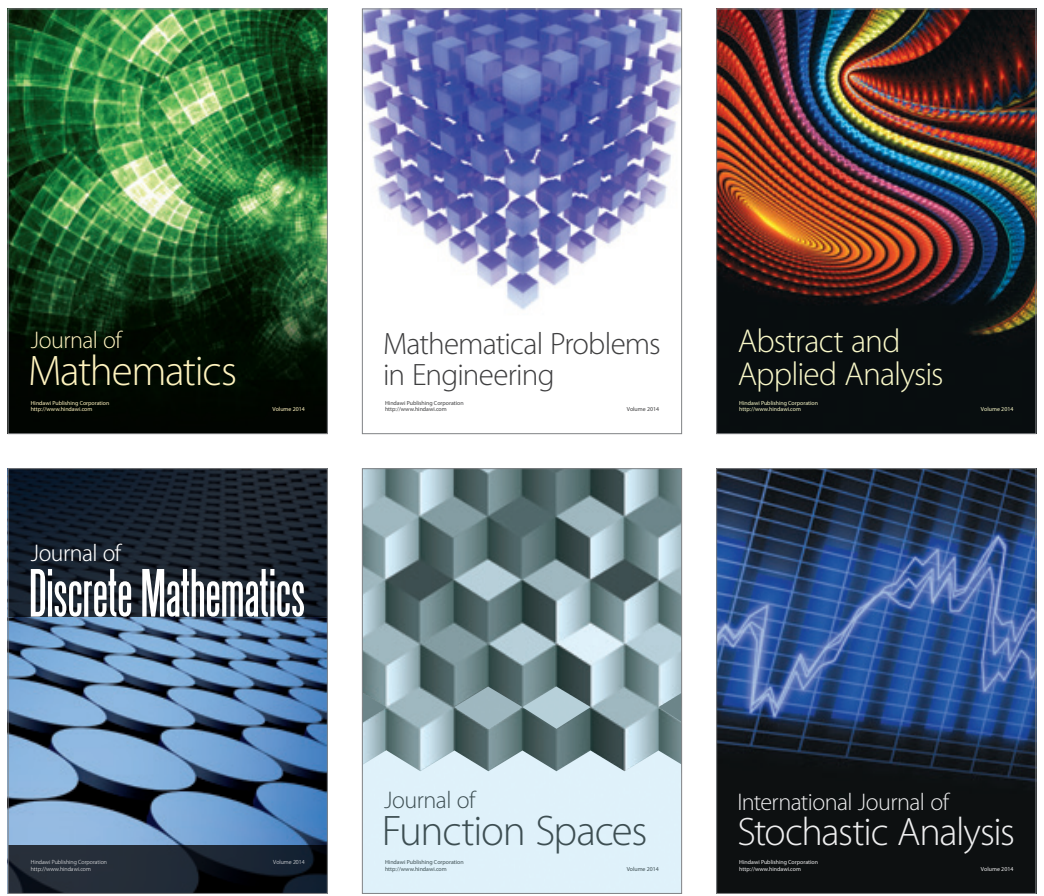

Journal of

Function Spaces

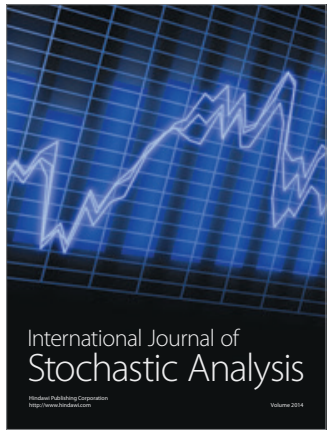

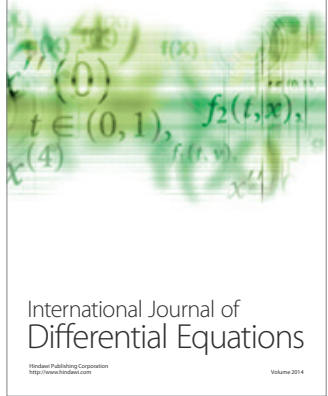
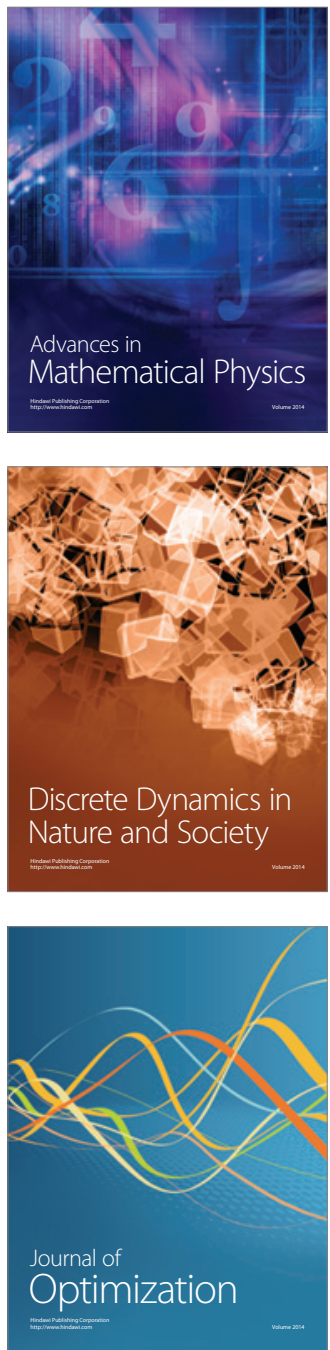